\title{
Dynamics Analysis of Avian Influenza A(H7N9) Epidemic Model
}

\author{
Yun Li, ${ }^{1}$ Peng Qin $\left(\mathbb{D},{ }^{2}\right.$ and Juping Zhang ${ }^{3}$ \\ ${ }^{1}$ School of Science, North University of China, Shanxi, Taiyuan 030051, China \\ ${ }^{2}$ School of Electrical and Control Engineering, North University of China, Shanxi, Taiyuan 030051, China \\ ${ }^{3}$ Complex Systems Research Center, Shanxi University, Taiyuan, Shanxi 030006, China
}

Correspondence should be addressed to Peng Qin; qinpeng@nuc.edu.cn

Received 31 January 2018; Accepted 2 April 2018; Published 23 May 2018

Academic Editor: J. R. Torregrosa

Copyright (C) 2018 Yun Li et al. This is an open access article distributed under the Creative Commons Attribution License, which permits unrestricted use, distribution, and reproduction in any medium, provided the original work is properly cited.

\begin{abstract}
The avian influenza $\mathrm{A}(\mathrm{H} 7 \mathrm{~N} 9)$ virus has certain fatal effects on human. In this paper, a mathematical model describing the transmission dynamics of avian influenza $\mathrm{A}(\mathrm{H} 7 \mathrm{~N} 9)$ between human and poultry is investigated. The basic reproduction number of the model is obtained by applying the method of the next generation matrix. Then the local and global stability of the equilibria are proven. At last, we use numerical simulations to verify the theoretical results.
\end{abstract}

\section{Introduction}

Infectious diseases are caused by a variety of bacteria, fungi, virus, and other pathogens, which can be transmitted between human and human, human and animals, animals and animals. Infectious diseases have always been the enemy of human; avian influenza is one of the most common diseases. According to the relevant studies, avian influenza was reported early in 1878 in Italy, which is caused by influenza A virus and is popular among animals. Meanwhile, it is transmitted to human by chickens, ducks, and other animals. The overwhelming majority of avian influenza virus cannot infect human; however, some avian influenza viruses are zoonotic [1]. The clinical manifestations of human diseases caused by some avian influenza virus are not very obvious, which are not harmful enough to human health. However, certain virus such as $\mathrm{H} 5 \mathrm{~N} 1$ and $\mathrm{H} 7 \mathrm{~N} 9$ can cause serious human diseases. The virus subtype H5N1, a highly pathogenic avian influenza virus, was firstly detected in Hong Kong in 1997 and was transmitted from Asia to Europe and Africa in 2003 and 2004, resulting in the death of a large amount of poultry and humans $[2,3]$. The virus subtype H7N9, a low pathogenic avian influenza virus, is transmitted mainly through contact. In March 2013, 3 people were firstly infected, and, by May 31, 132 cases were found, including 37 deaths, and the mortality rate even reached $30 \%$. These cases are distributed in some provinces such as Beijing, Shanghai,
Jiangsu, Zhejiang, Anhui, Shandong, Henan, Taiwan, and Fujian $[4,5]$. At present, infected humans of avian influenza A(H7N9) are still sporadic, and the ability of the virus to spread among humans has not yet been found. Since 2013, avian influenza $\mathrm{A}(\mathrm{H} 7 \mathrm{~N} 9)$ virus has been included in the main content of influenza virus research. The virus is not fatal to poultry, but it can cause severe respiratory diseases and certain lethality to human, which has attracted the attention of WHO [6].

At present, some scholars have studied avian influenza. In 2007, Iwami et al. [7] considered a dynamic model of avian influenza that might be transmitted by infected birds and infected humans with variant avian influenza. Che et al. [8] studied a model of highly pathogenic avian influenza with saturated contact rate. Initially, the health authorities controlled the outbreak of H7N9 by closing livepoultry markets, which can reduce the probability of human infected by reducing the contact between human and poultry. However, due to economic reasons, live-poultry markets cannot be permanently closed. Therefore, humans need to adopt other intervention strategies, such as screening poultry and killing infected poultry. Liu and Fang [9] established a dynamical model of avian influenza A(H7N9) that can spread between poultry and poultry, poultry and human, and human and human to evaluate the impact of these measures on avian influenza A(H7N9) epidemic. Chen and Wen [10] based on the bilinear disease incidence studied 
a model with mutant avian influenza $A(H 7 N 9)$ virus in 2015. In 2017, Liu et al. [11] proposed two avian influenza models with different growth rates of the avian population, one with logistic growth and the other with Allee effect.

The organization of this paper is as follows. In Section 2, consider that people mainly contact with poultry in wet markets. We construct an avian influenza A(H7N9) model of different groups in specific environment to study the transmission of avian influenza A(H7N9). In Sections 3 and 4 , the basic reproduction number and the existence of feasible equilibria are studied. What is more, by using suitable Lyapunov functions, we demonstrate the global stability of equilibria. The numerical simulations used to verify the theoretical results and some conclusions are included in Sections 5 and 6.

\section{The Model}

The exposure of infected poultry is one of the key factors in the human infection of avian influenza $\mathrm{A}(\mathrm{H} 7 \mathrm{~N} 9)$. In this section, we combine poultry with human to establish a mathematical model with the aim of understanding the spread of avian influenza A(H7N9) from poultry to human. Contagion occurs only between poultry and poultry as well as poultry and human; it cannot spread among humans. The human population is classified into three subclasses: susceptible, infected, and recovered, denoted by $S_{h}, I_{h}$, and $R_{h}$, respectively. $S_{f a}$ and $I_{f a}$ denote susceptible and infective poultry in farms and $S_{m a}$ and $I_{m a}$ represent susceptible poultry and infective poultry of markets, respectively. The flowchart of avian influenza A(H7N9) transmission between poultry and human is described in Figure 1.

The dynamic model of avian influenza $\mathrm{A}(\mathrm{H} 7 \mathrm{~N} 9)$ is described as the following ordinary differential equations:

$$
\begin{aligned}
\frac{d S_{f a}}{d t} & =A_{a}-d_{a} S_{f a}-a S_{f a}-\beta_{a} S_{f a} I_{f a}, \\
\frac{d I_{f a}}{d t} & =\beta_{a} S_{f a} I_{f a}-d_{a} I_{f a}-a I_{f a}-\alpha_{a} I_{f a}, \\
\frac{d S_{m a}}{d t} & =a S_{f a}-\beta_{m} S_{m a} I_{m a}-d_{m} S_{m a}, \\
\frac{d I_{m a}}{d t} & =a I_{f a}+\beta_{m} S_{m a} I_{m a}-d_{m} I_{m a}-\alpha_{m} I_{m a}, \\
\frac{d S_{h}}{d t} & =A_{h}-\beta_{h} S_{h} I_{m a}-d_{h} S_{h}, \\
\frac{d I_{h}}{d t} & =\beta_{h} S_{h} I_{m a}-d_{h} I_{h}-r I_{h}-\alpha_{h} I_{h}, \\
\frac{d R_{h}}{d t} & =r I_{h}-d_{h} R_{h},
\end{aligned}
$$

where $A_{h}$ and $A_{a}$ represent the birth rates of human and poultry, respectively. $d_{h}, d_{a}$, and $d_{m}$ indicate the natural mortality rates of human, poultry of farms, and poultry of markets, respectively. $\alpha_{h}, \alpha_{a}$, and $\alpha_{m}$ are the diseaserelated death rates of infected human, infected poultry of farms, and infected poultry of markets, respectively. $\beta_{a}$ is the transmission coefficient from infective poultry of farms to susceptible poultry of farms. $\beta_{m}$ is the contact rate from infective poultry of markets to susceptible poultry of markets. $\beta_{h}$ is the transmission rate from infected poultry of markets to susceptible human. $r$ is the recovery rate of infected human. $a$ is the proportion of poultry from farms to markets. All the parameters are nonnegative.

Let $S_{f a}(t)+I_{f a}(t)=N_{f a}(t), S_{m a}(t)+I_{m a}(t)=N_{m a}(t)$, $S_{h}(t)+I_{h}(t)+R_{h}(t)=N_{h}(t)$. From system (1), these can find that

$$
\begin{aligned}
\frac{d N_{f a}}{d t} & =A_{a}-\left(d_{a}+a\right) N_{f a}-\alpha_{a} I_{f a} \\
& \leq A_{a}-\left(d_{a}+a\right) N_{f a}, \\
\frac{d N_{m a}}{d t} & =a N_{f a}-d_{m} N_{m a}-\alpha_{m} I_{m a} \leq a N_{f a}-d_{m} N_{m a}, \\
\frac{d N_{h}}{d t} & =A_{h}-d_{a} N_{h}-\alpha_{h} I_{h} \leq A_{h}-d_{h} N_{h} .
\end{aligned}
$$

Then, from (2), it follows that

$$
N_{f a}(t) \leq \frac{A_{a}}{a+d_{a}}+\left(N_{f a}(0)-\frac{A_{a}}{a+d_{a}}\right) e^{-\left(a+d_{a}\right) t}
$$

and $e^{-\left(a+d_{a}\right) t} \rightarrow 0$ as $t \rightarrow \infty$, so $\lim _{t \rightarrow \infty} N_{f a}(t) \leq A_{a} /\left(a+d_{a}\right)$.

In the same way, from (3), it can be obtained that $\lim _{t \rightarrow \infty} N_{m a}(t) \leq a A_{a} / d_{m}\left(a+d_{a}\right), \lim _{t \rightarrow \infty} N_{h}(t) \leq A_{h} / d_{h}$. The feasible region of system (1) is

$$
\begin{aligned}
\Omega & =\left\{\left(S_{f a}, I_{f a}, S_{m a}, I_{m a}, S_{h}, I_{h}, R_{h}\right) \in R_{+}^{7}: N_{f a}\right. \\
& \left.\leq \frac{A_{a}}{a+d_{a}}, N_{m a} \leq \frac{a A_{a}}{d_{m}\left(a+d_{a}\right)}, \quad N_{h} \leq \frac{A_{h}}{d_{h}}\right\} .
\end{aligned}
$$

\section{The Existence of Equilibria}

By resolving these equations of system (1), it is easy to see that system (1) always has the disease-free equilibrium $E^{0}=$ $\left(S_{f a}^{0}, 0, S_{m a}^{0}, 0, S_{h}^{0}, 0,0\right)$, where

$$
\begin{aligned}
S_{f a}^{0} & =\frac{A_{a}}{d_{a}+a}, \\
S_{m a}^{0} & =\frac{a A_{a}}{d_{m}\left(d_{a}+a\right)}, \\
S_{h}^{0} & =\frac{A_{h}}{d_{h}} .
\end{aligned}
$$




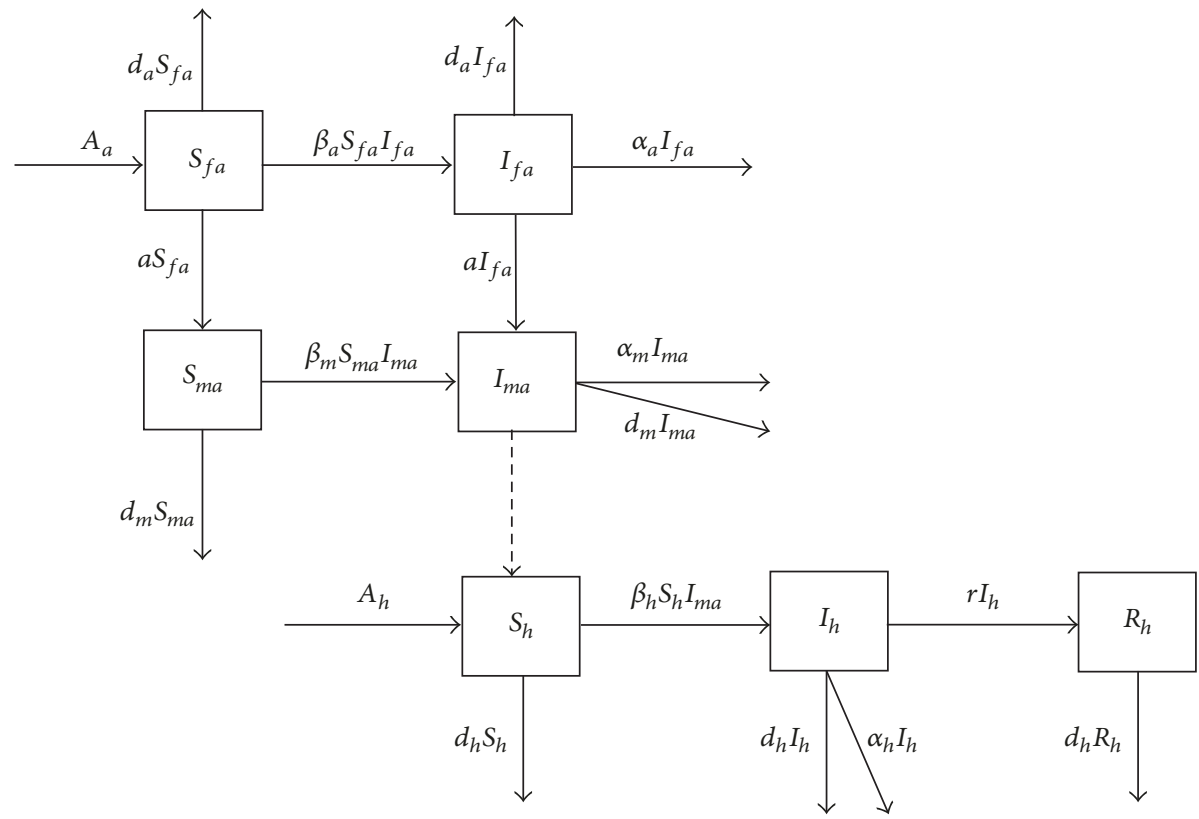

FIgURE 1: Flowchart of avian influenza A(H7N9) transmission.

According to the next generation matrix formulated in van den Driessche and Watmough [12], we can obtain

$$
\begin{aligned}
& F=\left(\begin{array}{ccc}
\frac{\beta_{a} A_{a}}{a+d_{a}} & 0 & 0 \\
0 & \frac{\beta_{m} a A_{a}}{d_{m}\left(a+d_{a}\right)} & 0 \\
0 & \frac{\beta_{h} A_{h}}{d_{h}} & 0
\end{array}\right), \\
& V=\left(\begin{array}{ccc}
\alpha_{a}+a+d_{a} & 0 & 0 \\
-a & d_{m}+\alpha_{m} & 0 \\
0 & 0 & d_{h}+\alpha_{h}+r
\end{array}\right) .
\end{aligned}
$$

Then

$$
\begin{aligned}
& R_{01}=\frac{\beta_{a} A_{a}}{\left(a+d_{a}+\alpha_{a}\right)\left(a+d_{a}\right)}, \\
& R_{02}=\frac{\beta_{m} a A_{a}}{d_{m}\left(a+d_{a}\right)\left(d_{m}+\alpha_{m}\right)} .
\end{aligned}
$$

Hence, the basic reproduction number of system is as follows:

$$
R_{0}=\max \left\{R_{01}, R_{02}\right\} .
$$

Theorem 1. The disease-free equilibrium $E^{0}$ of system (1) always exists. If $R_{0}<1, E^{0}$ is locally asymptotically stable; if $R_{0}>1$, it is unstable.

Proof. The Jacobian matrix at the disease-free equilibrium $E^{0}$ is

$$
J_{\mid E^{0}}=\left(\begin{array}{ccccccc}
-d_{a}-a & -\beta_{a} S_{f a}^{0} & 0 & 0 & 0 & 0 & 0 \\
0 & \beta_{a} S_{f a}^{0}-d_{a}-a-\alpha_{a} & 0 & 0 & 0 & 0 & 0 \\
a & 0 & -d_{m} & -\beta_{m} S_{m a}^{0} & 0 & 0 & 0 \\
0 & a & 0 & \beta_{m} S_{m a}^{0}-d_{m}-\alpha_{m} & 0 & 0 & 0 \\
0 & 0 & 0 & -\beta_{h} S_{h}^{0} & -d_{h} & 0 & 0 \\
0 & 0 & 0 & \beta_{h} S_{h}^{0} & 0 & -d_{h}-\alpha_{h}-r & 0 \\
0 & 0 & 0 & 0 & 0 & r & -d_{h}
\end{array}\right) .
$$


The characteristic equation of the Jacobian matrix is

$$
\begin{aligned}
& {\left[\left(\lambda+d_{a}+a\right)\left(\lambda+d_{a}+a+\alpha_{a}-\beta_{a} S_{f a}^{0}\right)\right]} \\
& \cdot\left[\left(\lambda+d_{m}\right)\left(\lambda+\alpha_{m}+d_{m}-\beta_{m} S_{m a}^{0}\right)\right] \\
& \cdot\left[\left(\lambda+d_{h}\right)\left(\lambda+d_{h}+r+\alpha_{h}\right)\left(\lambda+d_{h}\right)\right]=0 .
\end{aligned}
$$

The eigenvalues of the characteristic equation are

$$
\begin{aligned}
& \lambda_{1}=-d_{h}, \\
& \lambda_{2}=-d_{h}-r-\alpha_{h}, \\
& \lambda_{3}=-d_{h}, \\
& \lambda_{4}=-d_{a}-a, \\
& \lambda_{5}=-d_{m}, \\
& \lambda_{6}=-\alpha_{a}-a-d_{a}+\frac{\beta_{a} A_{a}}{a+d_{a}}, \\
& \lambda_{7}=-d_{m}-\alpha_{m}+\frac{\beta_{m} a A_{a}}{d_{m}\left(a+d_{a}\right)} .
\end{aligned}
$$

Hence, if $R_{01}<1$ and $R_{02}<1$, all eigenvalues have negative real parts. Namely, if $R_{0}<1$, the disease-free equilibrium $E^{0}$ is locally asymptotically stable.

The discussion of the existence of positive equilibrium is as follows.

(1) Let $I_{f a} \neq 0$; solving the second equation of system (1), one obtains that

$$
S_{f a}^{* *}=\frac{a+\alpha_{a}+d_{a}}{\beta_{a}} .
$$

Equation (13) is replaced with the first equation of system (1), which can be solved as follows:

$$
\begin{aligned}
I_{f a}^{* *} & =\frac{A_{a}-d_{a} S_{f a}^{* *}-a S_{f a}^{* *}}{\beta_{a} S_{f a}^{* *}} \\
& =\frac{\beta_{a} A_{a}-\left(a+d_{a}+\alpha_{a}\right)\left(d_{a}+a\right)}{\beta_{a}\left(a+d_{a}+\alpha_{a}\right)} \\
& =\frac{\left(a+d_{a}\right)\left(R_{01}-1\right)}{\beta_{a}} .
\end{aligned}
$$

Again, let $I_{m a}=0$; the fourth equation of system (1) appears as follows: its left is equal to $a I_{f a}^{* *}$, but the right is 0 . Both sides are not equal, so there is no $I_{m a}=0$. Let $I_{m a} \neq 0$, the third equation of system (1) can be solved as follows:

$$
S_{m a}^{* *}=\frac{a S_{f a}^{* *}}{\beta_{m} I_{m a}^{* *}+d_{m}} .
$$

Substituting (15) into the fourth equation of system (1) gives

$$
\begin{aligned}
& \beta_{m} S_{m a}^{* *} I_{m a}^{* *}+a I_{f a}^{* *}-d_{m} I_{m a}^{* *}-\alpha_{m} I_{m a}^{* *} \\
&= a I_{f a}^{* *}\left(\beta_{m} I_{m a}^{* *}+d_{m}\right) \\
& \quad+\left[a \beta_{m} S_{f a}^{* *}-\left(d_{m}+\alpha_{m}\right)\left(d_{m}+\beta_{m} I_{m a}^{* *}\right)\right] I_{m a}^{* *} \\
&=0
\end{aligned}
$$

and then

$$
a_{1} I_{m a}^{* * 2}+b_{1} I_{m a}^{* *}+c_{1}=0
$$

where

$$
\begin{aligned}
& a_{1}=-\beta_{m}\left(d_{m}+\alpha_{m}\right), \\
& b_{1}=a \beta_{m} I_{f a}^{* *}+a \beta_{m} S_{f a}^{* *}-d_{m}^{2}-d_{m} \alpha_{m}, \\
& c_{1}=a d_{m} I_{f a}^{* *} .
\end{aligned}
$$

Due to $a_{1}<0, c_{1}>0, \Delta=b_{1}^{2}-4 a_{1} c_{1}>0$ is always constant and there is a unique positive root. From (17), we obtain

$$
I_{m a}^{* *}=\frac{-a I_{f a}^{* *}}{\beta_{m} S_{m a}^{* *}-d_{m}-\alpha_{m}}=\frac{-b_{1}+\sqrt{b_{1}^{2}-4 a_{1} c_{1}}}{2 a_{1}} .
$$

Substituting (19) into the fifth, sixth, and seventh equations of system (1) gives

$$
\begin{aligned}
& S_{h}^{* *}=\frac{A_{h}}{\beta_{h} I_{m a}^{* *}+d_{h}}, \\
& I_{h}^{* *}=\frac{\beta_{h} S_{h}^{* *} I_{m a}^{* *}}{d_{h}+r+\alpha_{h}}, \\
& R_{h}^{* *}=\frac{r I_{h}^{* *}}{d_{h}} .
\end{aligned}
$$

(2) Let $I_{f a}=0$, from the first equation of (1), we can obtain

$$
S_{f a}^{*}=\frac{A_{a}}{d_{a}+a} .
$$

Again, let $I_{m a} \neq 0$. Substituting $I_{f a}=0$ into the fourth equation in (1) gives

$$
S_{m a}^{*}=\frac{d_{m}+\alpha_{m}}{\beta_{m}}
$$

Combining (21) and (22), from the third equation of system (1), it is known that

$$
\begin{aligned}
I_{m a}^{*} & =\frac{a S_{f a}^{*}-d_{m} S_{m a}^{*}}{\beta_{m} S_{m a}^{*}}=\frac{a A_{a}}{\left(d_{m}+\alpha_{m}\right)\left(a+d_{a}\right)}-\frac{d_{m}}{\beta_{m}} \\
& =\frac{d_{m}\left(R_{02}-1\right)}{\beta_{m}} .
\end{aligned}
$$


Substituting (23) into the fifth, sixth, and seventh equations of system (1) gives

$$
\begin{aligned}
S_{h}^{*} & =\frac{A_{h}}{\beta_{h} I_{m a}^{*}+d_{h}}, \\
I_{h}^{*} & =\frac{\beta_{h} S_{h}^{*} I_{m a}^{*}}{d_{h}+r+\alpha_{h}}, \\
R_{h}^{*} & =\frac{r I_{h}^{*}}{d_{h}} .
\end{aligned}
$$

Hence,

(i) when $R_{01}<1$, there are two cases:

when $R_{02}<1$, the disease-free equilibrium $E^{0}=$ $\left(S_{f a}^{0}, 0, S_{m a}^{0}, 0, S_{h}^{0}, 0,0\right)$ is obtained,

when $R_{02}>1$, the boundary equilibrium $E^{*}=$ $\left(S_{f a}^{*}, 0, S_{m a}^{*}, I_{m a}^{*}, S_{h}^{*}, I_{h}^{*}, R_{h}^{*}\right)$ is obtained;

(ii) when $R_{01}>1$, there are two cases:

when $R_{02}<1$, the positive equilibrium does not exist, when $R_{02}>1$, the endemic equilibrium $E^{* *}=$ $\left(S_{f a}^{* *}, I_{f a}^{* *}, S_{m a}^{* *}, I_{m a}^{* *}, S_{h}^{* *}, I_{h}^{* *}, R_{h}^{* *}\right)$ is obtained.

In conclusion, we can obtain the following theorem.

Theorem 2. For system (1), if $R_{01}<1, R_{02}>1$, there is the unique boundary equilibrium $E^{*}$; if $R_{01}>1, R_{02}>1$, there is the unique endemic equilibrium $E^{* *}$.

\section{Stability of Equilibria}

We note that the variable $R_{h}$ does not appear in the first six equations of system (1). The last equation is independent of the first six equations; we can only consider the following subsystem of system (1):

$$
\begin{aligned}
\frac{d S_{f a}}{d t} & =A_{a}-d_{a} S_{f a}-a S_{f a}-\beta_{a} S_{f a} I_{f a}, \\
\frac{d I_{f a}}{d t} & =\beta_{a} S_{f a} I_{f a}-d_{a} I_{f a}-a I_{f a}-\alpha_{a} I_{f a}, \\
\frac{d S_{m a}}{d t} & =a S_{f a}-\beta_{m} S_{m a} I_{m a}-d_{m} S_{m a}, \\
\frac{d I_{m a}}{d t} & =a I_{f a}+\beta_{m} S_{m a} I_{m a}-d_{m} I_{m a}-\alpha_{m} I_{m a}, \\
\frac{d S_{h}}{d t} & =A_{h}-\beta_{h} S_{h} I_{m a}-d_{h} S_{h}, \\
\frac{d I_{h}}{d t} & =\beta_{h} S_{h} I_{m a}-d_{h} I_{h}-r I_{h}-\alpha_{h} I_{h} .
\end{aligned}
$$

4.1. Stability of the Disease-Free Equilibrium. The discussion of global stability of the disease-free equilibrium $E^{0}$ is as follows.
The poultry subsystem of farms, the poultry subsystem of markets, and the human subsystem are independent of each other. We firstly consider the poultry subsystem in farms and define a Lyapunov function

$$
V_{11}=S_{f a}-S_{f a}^{0}-S_{f a}^{0} \ln \frac{S_{f a}}{S_{f a}^{0}}+I_{f a},
$$

and then the derivative of $V_{11}$ along solutions of system (25) is

$$
\begin{aligned}
\frac{d V_{11}}{d t}= & \frac{S_{f a}-S_{f a}^{0}}{S_{f a}} \frac{d S_{f a}}{d t}+\frac{d I_{f a}}{d t} \\
= & \frac{S_{f a}-S_{f a}^{0}}{S_{f a}}\left(A_{a}-d_{a} S_{f a}-a S_{f a}-\beta_{a} S_{f a} I_{f a}\right) \\
& +\beta_{a} S_{f a} I_{f a}-d_{a} I_{f a}-a I_{f a}-\alpha_{a} I_{f a} \\
= & \frac{S_{f a}-S_{f a}^{0}}{S_{f a}}\left(d_{a}\left(S_{f a}^{0}-S_{f a}\right)+a\left(S_{f a}^{0}-S_{f a}\right)\right) \\
& +\beta_{a} S_{f a}^{0} I_{f a}-I_{f a}\left(d_{a}+a+\alpha_{a}\right) \\
= & -\frac{a+d_{a}}{S_{f a}}\left(S_{f a}-S_{f a}^{0}\right)^{2} \\
& +\left(a+d_{a}+\alpha_{a}\right)\left(R_{01}-1\right) I_{f a},
\end{aligned}
$$

and if $R_{0}<1$, we get $d V_{11} / d t \leq 0$. Thus,

$\Omega_{1}=\left\{\left(S_{f a}, I_{f a}\right) \in R_{+}^{2}: d V_{11} / d t=0\right\}=\left\{\left(S_{f a}, I_{f a}\right) \in R_{+}^{2}:\right.$ $\left.S_{f a}=S_{f a}^{0}, I_{f a}=0\right\}=\left\{E_{f a}^{0}\right\}$. According to Lasalle's invariance principle [13,14], $E_{f a}^{0}$ is globally asymptotically stable.

Next, considering the poultry subsystem of markets with the avian components of farms already at the disease-free steady state

$$
\begin{aligned}
& \frac{d S_{m a}}{d t}=a S_{f a}^{0}-\beta_{m} S_{m a} I_{m a}-d_{m} S_{m a}, \\
& \frac{d I_{m a}}{d t}=\beta_{m} S_{m a} I_{m a}-d_{m} I_{m a}-\alpha_{m} I_{m a} .
\end{aligned}
$$

We define a Lyapunov function

$$
V_{12}=S_{m a}-S_{m a}^{0}-S_{m a}^{0} \ln \frac{S_{m a}}{S_{m a}^{0}}+I_{m a} .
$$

Calculating the derivative of $V_{12}$ along solutions of system (28), it follows that

$$
\begin{aligned}
\frac{d V_{12}}{d t}= & \frac{S_{m a}-S_{m a}^{0}}{S_{m a}} \frac{d S_{m a}}{d t}+\frac{d I_{m a}}{d t} \\
= & \frac{S_{m a}-S_{m a}^{0}}{S_{m a}}\left(a S_{f a}^{0}-d_{m} S_{m a}-\beta_{m} S_{m a} I_{m a}\right) \\
& +\beta_{m} S_{m a} I_{m a}-d_{m} I_{m a}-\alpha_{m} I_{m a}
\end{aligned}
$$




$$
\begin{aligned}
= & \frac{S_{m a}-S_{m a}^{0}}{S_{m a}}\left(d_{m}\left(S_{m a}^{0}-S_{m a}\right)\right)+\beta_{m} S_{m a}^{0} I_{m a} \\
& -d_{m} I_{m a}-\alpha_{m} I_{m a} \\
= & -\frac{d_{m}}{S_{m a}}\left(S_{m a}-S_{m a}^{0}\right)^{2} \\
& +\left(d_{m}+\alpha_{m}\right)\left(R_{02}-1\right) I_{m a},
\end{aligned}
$$

and if $R_{0}<1, d V_{12} / d t_{\mid(28)} \leq 0$. Thus, $\Omega_{2}=\left\{\left(S_{m a}, I_{m a}\right) \in R_{+}^{2}\right.$ : $\left.d V_{12} / d t=0\right\}=\left\{\left(S_{m a}, I_{m a}\right) \in R_{+}^{2}: S_{m a}=S_{m a}^{0}, I_{m a}=0\right\}=$ $\left\{E_{m a}^{0}\right\}$. According to Lasalle's invariance principle $[13,14], E_{m a}^{0}$ is globally asymptotically stable.

Finally, considering the human subsystem with the avian components already at the disease-free steady state

$$
\begin{aligned}
& \frac{d S_{h}}{d t}=A_{h}-d_{h} S_{h}, \\
& \frac{d I_{h}}{d t}=-d_{h} I_{h}-r I_{h}-\alpha_{h} I_{h} .
\end{aligned}
$$

We define a Lyapunov function

$$
V_{13}=S_{h}-S_{h}^{0}-S_{h}^{0} \ln \frac{S_{h}}{S_{h}^{0}}+I_{h},
$$

and then the derivative of $V_{13}$ along solutions of system (31) is

$$
\begin{aligned}
\frac{d V_{13}}{d t} & =\frac{S_{h}-S_{h}^{0}}{S_{h}} \frac{d S_{h}}{d t}+\frac{d I_{h}}{d t} \\
& =\frac{S_{h}-S_{h}^{0}}{S_{h}}\left(A_{h}-d_{h} S_{h}\right)-d_{h} I_{h}-r I_{h}-\alpha_{h} I_{h} \\
& =-\frac{d_{h}}{S_{h}}\left(S_{h}-S_{h}^{0}\right)^{2}-\left(r+d_{h}+\alpha_{h}\right) I_{h} \leq 0,
\end{aligned}
$$

and, thus, $\Omega_{3}=\left\{\left(S_{h}, I_{h}\right) \in R_{+}^{2}: d V_{13} / d t=0\right\}=\left\{\left(S_{h}, I_{h}\right) \in\right.$ $\left.R_{+}^{2}: S_{h}=S_{h}^{0}, I_{h}=0\right\}=\left\{E_{h}^{0}\right\}$. According to Lasalle's invariance principle $[13,14], E_{h}^{0}$ is globally asymptotically stable. In summary, the following theorem can be obtained.

Theorem 3. For system (1), if $R_{0}<1$, the disease-free equilibrium $E^{0}$ is globally asymptotically stable.

4.2. Stability of the Boundary Equilibrium and the Endemic Equilibrium. After calculation, the Jacobian matrix of system (1) is given as

$$
J=\left(\begin{array}{cc}
G & 0 \\
H & K
\end{array}\right)
$$

where

$$
\begin{aligned}
G & =\left(\begin{array}{cccc}
-d_{a}-a-\beta_{a} I_{f a} & -\beta_{a} S_{f a} & 0 & 0 \\
\beta_{a} I_{f a} & \beta_{a} S_{f a}-d_{a}-a-\alpha_{a} & 0 & 0 \\
a & 0 & -\beta_{m} I_{m a}-d_{m} & -\beta_{m} S_{m a} \\
0 & a & \beta_{m} I_{m a} & \beta_{m} S_{m a}-d_{m}-\alpha_{m}
\end{array}\right), \\
H & =\left(\begin{array}{cccc}
0 & 0 & 0 & -\beta_{h} S_{h} \\
0 & 0 & 0 & \beta_{h} S_{h} \\
0 & 0 & 0 & 0
\end{array}\right), \\
K & =\left(\begin{array}{ccc}
-\beta_{h} I_{m a}-d_{h} & 0 & 0 \\
\beta_{h} I_{m a} & -d_{h}-r-\alpha_{h} & 0 \\
0 & r & -d_{h}
\end{array}\right) .
\end{aligned}
$$

The characteristic equation of the Jacobian matrix is

$$
\begin{aligned}
& {\left[\left(\lambda+d_{a}+a+\beta_{a} I_{f a}\right)\left(\lambda+d_{a}+a+\alpha_{a}-\beta_{a} S_{f a}\right)\right.} \\
& \left.\quad+\beta_{a}^{2} S_{f a} I_{f a}\right] \\
& \quad \cdot\left[\left(\lambda+d_{m}+\beta_{m} I_{m a}\right)\left(\lambda+\alpha_{m}+d_{m}-\beta_{m} S_{m a}\right)\right. \\
& \left.\quad+\beta_{m}^{2} S_{m a} I_{m a}\right] \\
& \quad \cdot\left[\left(\lambda+d_{h}+\beta_{h} I_{m a}\right)\left(\lambda+d_{h}+r+\alpha_{h}\right)\left(\lambda+d_{h}\right)\right] \\
& \quad=0 .
\end{aligned}
$$

(1) For the boundary equilibrium $E^{*}=\left(S_{f a}^{*}, 0, S_{m a}^{*}, I_{m a}^{*}, S_{h}^{*}\right.$, $I_{h}^{*}, R_{h}^{*}$ ), five eigenvalues are

$$
\begin{aligned}
& \lambda_{1}=-d_{h}, \\
& \lambda_{2}=-d_{h}-r-\alpha_{h}, \\
& \lambda_{3}=-d_{h}-\beta_{h} I_{m a}^{*}, \\
& \lambda_{4}=-a-d_{a}, \\
& \lambda_{5}=-\alpha_{a}-a-d_{a}+\frac{\beta_{a} A_{a}}{a+d_{a}} .
\end{aligned}
$$


The remaining two eigenvalues $\lambda_{6}, \lambda_{7}$ depend on

$$
\lambda^{2}+\lambda d_{m}\left(R_{02}-1\right)+d_{m}\left(\alpha_{m}+d_{m}\right)\left(R_{02}-1\right)=0 .
$$

Hence, if $R_{01}<1, R_{02}>1$, all eigenvalues have negative real parts.

(2) For the endemic equilibrium $E^{* *}=\left(S_{f a}^{* *}, I_{f a}^{* *}, S_{m a}^{* *}\right.$, $\left.I_{m a}^{* *}, S_{h}^{* *}, I_{h}^{* *}, R_{h}^{* *}\right)$, three eigenvalues are

$$
\begin{aligned}
& \lambda_{1}=-d_{h}, \\
& \lambda_{2}=-d_{h}-r-\alpha_{h}, \\
& \lambda_{3}=-d_{h}-\beta_{h} I_{m a}^{* *} .
\end{aligned}
$$

In addition, $\lambda_{4}, \lambda_{5}$ are satisfied:

$$
\begin{aligned}
\lambda^{2} & +\lambda R_{01}\left(d_{a}+a\right)+\left(d_{a}+a\right)\left(\alpha+a+d_{a}\right)\left(R_{01}-1\right) \\
& =0 .
\end{aligned}
$$

The remaining two eigenvalues $\lambda_{6}, \lambda_{7}$ are satisfied:

$$
\begin{aligned}
\lambda^{2}+ & \lambda\left(2 d_{m}+\alpha_{m}-\beta_{m} S_{m a}^{* *}+\beta_{m} I_{m a}^{* *}\right)+\beta_{m} d_{m} I_{m a}^{* *} \\
+ & \beta_{m} \alpha_{m} I_{m a}^{* *}+d_{m}\left(d_{m}+\alpha_{m}-\beta_{m} S_{m a}^{* *}\right)=0
\end{aligned}
$$

It can be obtained that $d_{m}+\alpha_{m}-\beta_{m} S_{m a}^{* *}>0$ by $I_{m a}^{* *}>0$. Hence, if $R_{01}>1, R_{02}>1$, all eigenvalues have negative real parts. According to the above discussion, we can derive the following theorem.

Theorem 4. For system (1), if $R_{01}<1, R_{02}>1$, the boundary equilibrium $E^{*}$ is locally asymptotically stable; if $R_{01}>1, R_{02}>$ 1 , the endemic equilibrium $E^{* *}$ is locally asymptotically stable.

Consider the global stability of the boundary equilibrium and the endemic equilibrium.

(1) If $R_{01}<1, R_{02}>1$, there is the boundary equilibrium $E^{*}$.

We firstly consider the poultry subsystem in farms and define a Lyapunov function

$$
V_{21}=S_{f a}-S_{f a}^{*}-S_{f a}^{*} \ln \frac{S_{f a}}{S_{f a}^{*}}+I_{f a}
$$

and then the derivative of $V_{21}$ along solutions of system (25) is

$$
\begin{aligned}
\frac{d V_{21}}{d t}= & \frac{S_{f a}-S_{f a}^{*}}{S_{f a}} \frac{d S_{f a}}{d t}+\frac{d I_{f a}}{d t} \\
= & \frac{S_{f a}-S_{f a}^{*}}{S_{f a}}\left(A_{a}-d_{a} S_{f a}-a S_{f a}-\beta_{a} S_{f a} I_{f a}\right) \\
& +\beta_{a} S_{f a} I_{f a}-d_{a} I_{f a}-a I_{f a}-\alpha_{a} I_{f a} \\
= & \frac{S_{f a}-S_{f a}^{*}}{S_{f a}}\left(d_{a}\left(S_{f a}^{*}-S_{f a}\right)+a\left(S_{f a}^{*}-S_{f a}\right)\right)
\end{aligned}
$$

$$
\begin{aligned}
& +\beta_{a} S_{f a}^{*} I_{f a}-I_{f a}\left(d_{a}+a+\alpha_{a}\right) \\
= & -\frac{a+d_{a}}{S_{f a}}\left(S_{f a}-S_{f a}^{*}\right)^{2} \\
& +\left(a+d_{a}+\alpha_{a}\right)\left(R_{01}-1\right) I_{f a},
\end{aligned}
$$

and if $R_{01}<1, d V_{21} / d t \leq 0$, thus $\Omega_{4}=\left\{\left(S_{f a}, I_{f a}\right) \in R_{+}^{2}\right.$ : $\left.d V_{21} / d t=0\right\}=\left\{\left(S_{f a}, I_{f a}\right) \in R_{+}^{2}: S_{f a}=S_{f a}^{*}, I_{f a}=0\right\}=$ $\left\{E_{f a}^{*}\right\}$. According to Lasalle's invariance principle $[13,14], E_{f a}^{*}$ is globally asymptotically stable.

Next, considering the poultry subsystem of markets with the avian components of farms already at the disease-free steady state

$$
\begin{aligned}
& \frac{d S_{m a}}{d t}=a S_{f a}^{*}-\beta_{m} S_{m a} I_{m a}-d_{m} S_{m a} \\
& \frac{d I_{m a}}{d t}=\beta_{m} S_{m a} I_{m a}-d_{m} I_{m a}-\alpha_{m} I_{m a} .
\end{aligned}
$$

We define a Lyapunov function

$$
\begin{aligned}
V_{22}= & S_{m a}-S_{m a}^{*}-S_{m a}^{*} \ln \frac{S_{m a}}{S_{m a}^{*}}+I_{m a}-I_{m a}^{*} \\
& -I_{m a}^{*} \ln \frac{I_{m a}}{I_{m a}^{*}}
\end{aligned}
$$

and then the derivative of $V_{22}$ along solutions of system (44) is

$$
\begin{aligned}
& \frac{d V_{22}}{d t}=\frac{S_{m a}-S_{m a}^{*}}{S_{m a}} \frac{d S_{m a}}{d t}+\frac{I_{m a}-I_{m a}^{*}}{I_{m a}} \frac{d I_{m a}}{d t} \\
& =\frac{S_{m a}-S_{m a}^{*}}{S_{m a}}\left(a S_{f a}^{*}-d_{m} S_{m a}-\beta_{m} S_{m a} I_{m a}\right) \\
& +\frac{I_{m a}-I_{m a}^{*}}{I_{m a}}\left(\beta_{m} S_{m a} I_{m a}-d_{m} I_{m a}-\alpha_{m} I_{m a}\right) \\
& =\frac{S_{m a}-S_{m a}^{*}}{S_{m a}}\left(d_{m}\left(S_{m a}^{*}-S_{m a}\right)+\beta_{m} S_{m a}^{*} I_{m a}^{*}\right. \\
& \left.-\beta_{m} S_{m a} I_{m a}\right)+\frac{I_{m a}-I_{m a}^{*}}{I_{m a}}\left(\beta_{m} S_{m a} I_{m a}\right. \\
& \left.-\beta_{m} S_{m a}^{*} I_{m a}\right)=\left(2-\frac{S_{m a}^{*}}{S_{m a}}-\frac{S_{m a}}{S_{m a}^{*}}\right)\left(d_{m} S_{m a}^{*}\right. \\
& \left.+\beta_{m} S_{m a}^{*} I_{m a}^{*}\right)=\left(2-\frac{S_{m a}^{*}}{S_{m a}}-\frac{S_{m a}}{S_{m a}^{*}}\right)\left(d_{m} S_{m a}^{*}\right. \\
& \left.+S_{m a}^{*} d_{m}\left(R_{02}-1\right)\right) .
\end{aligned}
$$

Since $2-S_{m a}^{*} / S_{m a}-S_{m a} / S_{m a}^{*} \leq 0$, if $R_{02}>1, d V_{22} / d t_{\mid(44)} \leq$ 0 , thus, $\Omega_{5}=\left\{\left(S_{m a}, I_{m a}\right) \in R_{+}^{2}: d V_{22} / d t=0\right\}=\left\{\left(S_{m a}, I_{m a}\right) \in\right.$ $\left.R_{+}^{2}: S_{m a}=S_{m a}^{*}, I_{m a}=I_{m a}^{*}\right\}=\left\{E_{m a}^{*}\right\}$. According to Lasalle's invariance principle $[13,14], E_{m a}^{*}$ is globally asymptotically stable. 
Finally, considering the human subsystem with the avian components of markets already at the endemic steady state

$$
\begin{aligned}
& \frac{d S_{h}}{d t}=A_{h}-\beta_{h} S_{h} I_{m a}^{*}-d_{h} S_{h}, \\
& \frac{d I_{h}}{d t}=\beta_{h} S_{h} I_{m a}^{*}-d_{h} I_{h}-r I_{h}-\alpha_{h} I_{h} .
\end{aligned}
$$

We define a Lyapunov function

$$
V_{23}=S_{h}-S_{h}^{*}-S_{h}^{*} \ln \frac{S_{h}}{S_{h}^{*}}+I_{h}-I_{h}^{*}-I_{h}^{*} \ln \frac{I_{h}}{I_{h}^{*}},
$$

and then the derivative of $V_{23}$ along solutions of system (47) is

$$
\begin{aligned}
\frac{d V_{23}}{d t}= & \frac{S_{h}-S_{h}^{*}}{S_{h}} \frac{d S_{h}}{d t}+\frac{I_{h}-I_{h}^{*}}{I_{h}} \frac{d I_{h}}{d t} \\
= & \frac{S_{h}-S_{h}^{*}}{S_{h}}\left(A_{h}-\beta_{h} S_{h} I_{m a}^{*}-d_{h} S_{h}\right) \\
& +\frac{I_{h}-I_{h}^{*}}{I_{h}}\left(\beta_{h} S_{h} I_{m a}^{*}-d_{h} I_{h}-r I_{h}-\alpha_{h} I_{h}\right) \\
= & \frac{S_{h}-S_{h}^{*}}{S_{h}}\left(d_{h} S_{h}^{*}-d_{h} S_{h}+\beta_{h} S_{h}^{*} I_{m a}^{*}-\beta_{h} S_{h} I_{m a}^{*}\right) \\
& +\frac{I_{h}-I_{h}^{*}}{I_{h}}\left(\beta_{h} S_{h} I_{m a}^{*}-\beta_{h} S_{h}^{*} I_{m a}^{*} \frac{I_{h}}{I_{h}^{*}}\right) \\
= & d_{h} S_{h}^{*}\left(2-\frac{S_{h}^{*}}{S_{h}}-\frac{S_{h}}{S_{h}^{*}}\right) \\
& +\beta_{h} S_{h}^{*} I_{m a}^{*}\left(3-\frac{S_{h}^{*}}{S_{h}}-\frac{I_{h}}{I_{h}^{*}}-\frac{S_{h} I_{h}^{*}}{S_{h}^{*} I_{h}}\right) \\
= & d_{h} S_{h}^{*}\left(2-\frac{S_{h}^{*}}{S_{h}}-\frac{S_{h}}{S_{h}^{*}}\right) \\
& +\beta_{h} S_{h}^{*} \frac{d_{m}\left(R_{02}-1\right)}{\beta_{m}}\left(3-\frac{S_{h}^{*}}{S_{h}}-\frac{I_{h}}{I_{h}^{*}}-\frac{S_{h} I_{h}^{*}}{S_{h}^{*} I_{h}}\right) .
\end{aligned}
$$

Since $2-S_{h}^{*} / S_{h}-S_{h} / S_{h}^{*} \leq 0,3-S_{h}^{*} / S_{h}-I_{h} / I_{h}^{*}-S_{h} I_{h}^{*} / S_{h}^{*} I_{h} \leq 0$, if $R_{02}>1, d V_{23} / d t_{\mid(47)} \leq 0$, thus, $\Omega_{6}=\left\{\left(S_{h}, I_{h}\right) \in R_{+}^{2}: d V_{23} /\right.$ $d t=0\}=\left\{\left(S_{h}, I_{h}\right) \in R_{+}^{2}: S_{h}=S_{h}^{*}, I_{h}=I_{h}^{*}\right\}=\left\{E_{h}^{*}\right\}$. According to Lasalle's invariance principle $[13,14], E_{h}^{*}$ is globally asymptotically stable. In conclusion, if $R_{01}<1, R_{02}>1$, the boundary equilibrium $E^{*}$ is globally asymptotically stable. $E^{* *}$.

(2) If $R_{01}>1, R_{02}>1$, there is the endemic equilibrium

We firstly consider the poultry subsystem in farms and define a Lyapunov function

$$
\begin{aligned}
V_{31}= & S_{f a}-S_{f a}^{* *}-S_{f a}^{* *} \ln \frac{S_{f a}}{S_{f a}^{* *}}+I_{f a}-I_{f a}^{* *} \\
& -I_{f a}^{* *} \ln \frac{I_{f a}}{I_{f a}^{* *}},
\end{aligned}
$$

and then the derivative of $V_{31}$ along solutions of system (25) is

$$
\begin{aligned}
\frac{d V_{31}}{d t}= & \frac{S_{f a}-S_{f a}^{* *}}{S_{f a}} \frac{d S_{f a}}{d t}+\frac{I_{f a}-I_{f a}^{* *}}{I_{f a}} \frac{d I_{f a}}{d t} \\
= & \frac{S_{f a}-S_{f a}^{* *}}{S_{f a}}\left(A_{a}-d_{a} S_{f a}-a S_{f a}-\beta_{a} S_{f a} I_{f a}\right) \\
& +\frac{I_{f a}-I_{f a}^{* *}}{I_{f a}}\left(\beta_{a} S_{f a} I_{f a}-d_{a} I_{f a}-a I_{f a}-\alpha_{a} I_{f a}\right) \\
= & \frac{S_{f a}-S_{f a}^{* *}}{S_{f a}}\left[\left(d_{a}+a\right)\left(S_{f a}^{* *}-S_{f a}\right)\right]+\beta_{a} S_{f a}^{* *} I_{f a}^{* *} \\
& -\beta_{a} S_{f a} I_{f a}-\beta_{a} S_{f a}^{* *} I_{f a}^{* *} \frac{S_{f a}^{* *}}{S_{f a}}+\beta_{a} S_{f a}^{* *} I_{f a} \\
& +\beta_{a} S_{f a} I_{f a}-\beta_{a} S_{f a}^{* *} I_{f a}-\beta_{a} S_{f a} I_{f a}^{* *}+\beta_{a} S_{f a}^{* *} I_{f a}^{* *} \\
= & -\frac{a+d_{a}}{S_{f a}}\left(S_{f a}-S_{f a}^{* *}\right)^{2} \\
& +\beta_{a} S_{f a}^{* *} I_{f a}^{* *}\left(2-\frac{S_{f a}^{* *}}{S_{f a}}-\frac{S_{f a}}{S_{f a}^{* * *}}\right) \\
= & +\frac{a+d_{a}}{S_{f a}}\left(S_{f a}-S_{f a}^{* *}\right)^{2} \\
& S_{f a}^{* *}\left(a+d_{a}\right)\left(R_{01}-1\right)\left(2-\frac{S_{f a}^{* *}}{S_{f a}}-\frac{S_{f a}}{S_{f a}^{* *}}\right) .
\end{aligned}
$$

Since $2-S_{f a}^{* *} / S_{f a}-S_{f a} / S_{f a}^{* *} \leq 0$, if $R_{01}>1, d V_{31} / d t \leq 0$, thus, $\Omega_{7}=\left\{\left(S_{f a}, I_{f a}\right) \in R_{+}^{2}: d V_{31} / d t=0\right\}=\left\{\left(S_{f a}, I_{f a}\right) \in\right.$ $\left.R_{+}^{2}: S_{f a}=S_{f a}^{* *}, I_{f a}=I_{f a}^{* *}\right\}=\left\{E_{f a}^{* *}\right\}$. According to Lasalle's invariance principle $[13,14], E_{f a}^{* *}$ is globally asymptotically stable.

Next, considering the poultry subsystem of markets with the avian components of farms already at the endemic steady state

$$
\begin{aligned}
& \frac{d S_{m a}}{d t}=a S_{f a}^{* *}-\beta_{m} S_{m a} I_{m a}-d_{m} S_{m a} \\
& \frac{d I_{m a}}{d t}=a I_{f a}^{* *}+\beta_{m} S_{m a} I_{m a}-d_{m} I_{m a}-\alpha_{m} I_{m a} .
\end{aligned}
$$

We define a Lyapunov function

$$
\begin{aligned}
V_{32}= & S_{m a}-S_{m a}^{* *}-S_{m a}^{* *} \ln \frac{S_{m a}}{S_{m a}^{* *}}+I_{m a}-I_{m a}^{* *} \\
& -I_{m a}^{* *} \ln \frac{I_{m a}}{I_{m a}^{* * *}}
\end{aligned}
$$


and then the derivative of $V_{32}$ along solutions of system (52) is

$$
\begin{aligned}
& \frac{d V_{32}}{d t}=\frac{S_{m a}-S_{m a}^{* *}}{S_{m a}} \frac{d S_{m a}}{d t}+\frac{I_{m a}-I_{m a}^{* *}}{I_{m a}} \frac{d I_{m a}}{d t} \\
& =\frac{S_{m a}-S_{m a}^{* *}}{S_{m a}}\left(a S_{f a}^{* *}-d_{m} S_{m a}-\beta_{m} S_{m a} I_{m a}\right) \\
& +\frac{I_{m a}-I_{m a}^{* *}}{I_{m a}}\left(a I_{f a}^{* *}+\beta_{m} S_{m a} I_{m a}-d_{m} I_{m a}-\alpha_{m} I_{m a}\right) \\
& =\frac{S_{m a}-S_{m a}^{* *}}{S_{m a}}\left[d_{m}\left(S_{m a}^{* *}-S_{m a}\right)+\beta_{m} S_{m a}^{* *} I_{m a}^{* *}\right. \\
& \left.-\beta_{m} S_{m a} I_{m a}\right]+\frac{I_{m a}-I_{m a}^{* *}}{I_{m a}}\left(a I_{f a}^{* *}+\beta_{m} S_{m a} I_{m a}\right. \\
& \left.-\left(a I_{f a}^{* *}+\beta_{m} S_{m a}^{* *} I_{m a}^{* *}\right) \frac{I_{m a}}{I_{m a}^{* *}}\right) \\
& =\frac{a\left(a+d_{a}\right)\left(R_{01}-1\right)}{\beta_{a}}\left(2-\frac{I_{m a}^{* *}}{I_{m a}}-\frac{I_{m a}}{I_{m a}^{* *}}\right) \\
& +d_{m} S_{m a}^{* *}\left(2-\frac{S_{m a}^{* *}}{S_{m a}}-\frac{S_{m a}}{S_{m a}^{* * *}}\right)+\beta_{m} S_{m a}^{* *} I_{m a}^{* *}(2 \\
& \left.-\frac{S_{m a}^{* *}}{S_{m a}}-\frac{S_{m a}}{S_{m a}^{* *}}\right) .
\end{aligned}
$$

Since $2-S_{m a}^{* *} / S_{m a}-S_{m a} / S_{m a}^{* *} \leq 0,2-I_{m a}^{* *} / I_{m a}-I_{m a} / I_{m a}^{* *} \leq 0$, if $R_{01}>1, R_{02}>1, I_{m a}^{* *}>0, d V_{32} / d t_{\mid(52)} \leq 0$, thus, $\Omega_{8}=$ $\left\{\left(S_{m a}, I_{m a}\right) \in R_{+}^{2}: d V_{32} / d t=0\right\}=\left\{\left(S_{m a}, I_{m a}\right) \in R_{+}^{2}: S_{m a}=\right.$ $\left.S_{m a}^{* *}, I_{m a}=I_{m a}^{* *}\right\}=\left\{E_{m a}^{* *}\right\}$. According to Lasalle's invariance principle $[13,14], E_{m a}^{* *}$ is globally asymptotically stable.

Finally, considering the human subsystem with the avian components already at the endemic steady state

$$
\begin{aligned}
& \frac{d S_{h}}{d t}=A_{h}-\beta_{h} S_{h} I_{m a}^{* *}-d_{h} S_{h}, \\
& \frac{d I_{h}}{d t}=\beta_{h} S_{h} I_{m a}^{* *}-d_{h} I_{h}-r I_{h}-\alpha_{h} I_{h} .
\end{aligned}
$$

We define a Lyapunov function

$$
V_{33}=S_{h}-S_{h}^{* *}-S_{h}^{* *} \ln \frac{S_{h}}{S_{h}^{* *}}+I_{h}-I_{h}^{* *}-I_{h}^{* *} \ln \frac{I_{h}}{I_{h}^{* *}},
$$

and then the derivative of $V_{33}$ along solutions of system (55) is

$$
\begin{aligned}
\frac{d V_{33}}{d t} & =\frac{S_{h}-S_{h}^{* *}}{S_{h}} \frac{d S_{h}}{d t}+\frac{I_{h}-I_{h}^{* *}}{I_{h}} \frac{d I_{h}}{d t} \\
& =\frac{S_{h}-S_{h}^{* *}}{S_{h}}\left(A_{h}-\beta_{h} S_{h} I_{m a}^{* *}-d_{h} S_{h}\right)
\end{aligned}
$$

$$
\begin{aligned}
& +\frac{I_{h}-I_{h}^{* *}}{I_{h}}\left(\beta_{h} S_{h} I_{m a}^{* *}-d_{h} I_{h}-r I_{h}-\alpha_{h} I_{h}\right) \\
= & d_{h}\left(S_{h}^{* *}-S_{h}\right)+\beta_{h} S_{h}^{* *} I_{m a}^{* *}-\beta_{h} S_{h} I_{m a}^{* *} \\
& -\beta_{h} S_{h}^{* *} I_{m a}^{* *} \frac{S_{h}^{* *}}{S_{h}}-d_{h} S_{h}^{* *} \frac{S_{h}^{* *}}{S_{h}}+\beta_{h} S_{h}^{* *} I_{m a}^{* *} \\
& +d_{h} S_{h}^{* *}+\beta_{h} S_{h} I_{m a}^{* *}-\beta_{h} S_{h}^{* * *} I_{m a}^{* *} \frac{I_{h}}{I_{h}^{* *}} \\
& +\beta_{h} S_{h}^{* *} I_{m a}^{* *}-\beta_{h} S_{h} I_{m a}^{* *} \frac{I_{h}^{* *}}{I_{h}} \\
= & d_{h} S_{h}^{* *}\left(2-\frac{S_{h}^{* *}}{S_{h}}-\frac{S_{h}}{S_{h}^{* *}}\right) \\
& +\beta_{h} S_{h}^{* *} I_{m a}^{* *}\left(3-\frac{S_{h}^{* *}}{S_{h}}-\frac{I_{h}}{I_{h}^{* *}}-\frac{S_{h} I_{h}^{* *}}{S_{h}^{* *} I_{h}}\right) .
\end{aligned}
$$

Since $2-S_{h}^{* *} / S_{h}-S_{h} / S_{h}^{* *} \leq 0,3-S_{h}^{* *} / S_{h}-I_{h} / I_{h}^{* *}-$ $S_{h} I_{h}^{* *} / S_{h}^{* *} I_{h} \leq 0$, if $R_{02}>1, I_{m a}^{* *}>0, d V_{33} / d t_{\mid(55)} \leq 0$, thus, $\Omega_{9}=\left\{\left(S_{h}, I_{h}\right) \in R_{+}^{2}: d V_{33} / d t=0\right\}=\left\{\left(S_{h}, I_{h}\right) \in R_{+}^{2}: S_{h}=\right.$ $\left.S_{h}^{* *}, I_{h}=I_{h}^{* * *}\right\}=\left\{E_{h}^{* *}\right\}$. According to Lasalle's invariance principle $[13,14], E_{h}^{* *}$ is globally asymptotically stable. Hence, the following theorem can be obtained.

Theorem 5. For system (1), if $R_{01}<1, R_{02}>1$, the boundary equilibrium $E^{*}$ is globally asymptotically stable; if $R_{01}>1$, $R_{02}>1$, the endemic equilibrium $E^{* *}$ is globally asymptotically stable.

Remark 6. Stability of the equilibrium depends on the Lyapunov functions. The quadratic form of Lyapunov functions is usually used in most references $[15,16]$. Let us take the function in this paper

$$
f(x)=1-x+\ln x, \quad \forall x>0,
$$

we have

$$
\begin{aligned}
f(1) & =0, \\
f^{\prime}(x) & =\frac{1}{x}-1 ;
\end{aligned}
$$

thus, $f(x)=1-x+\ln x \leq 0$, and the equality holds only when $x=1$. Let $x=S_{f a} / S_{f a}^{0}>0$; then $S_{f a}-S_{f a}^{0}-$ $S_{f a}^{0} \ln \left(S_{f a} / S_{f a}^{0}\right) \geq 0$. Hence, $V_{11}$ is a Lyapunov function. In a similar way, $V_{12}, V_{13}, V_{21}, V_{22}, V_{23}, V_{31}, V_{32}, V_{33}$ are also Lyapunov functions.

\section{Numerical Simulations}

In this section, the following parameter values are taken as some examples to simulate the stability of the diseasefree equilibrium, the boundary equilibrium, and the endemic equilibrium of system (1), and the time-series diagram is given. At last, when $\beta_{h}$ has different parameter values, the time-variation diagram of $I_{h}$ is given. 

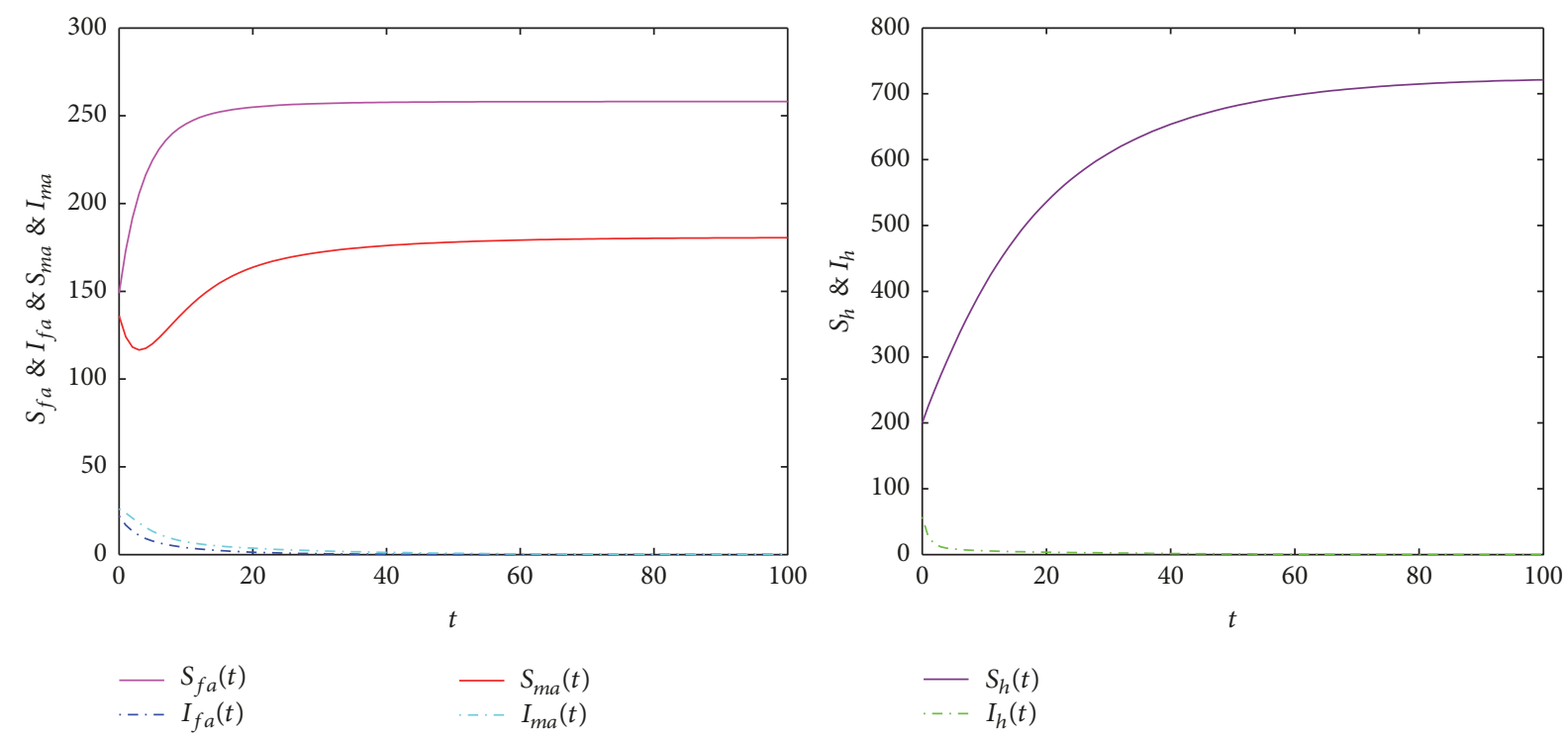

FIGURE 2: If $R_{0}=0.88680353<1$, the time-variation diagram of system $(25)$ state variables is shown.
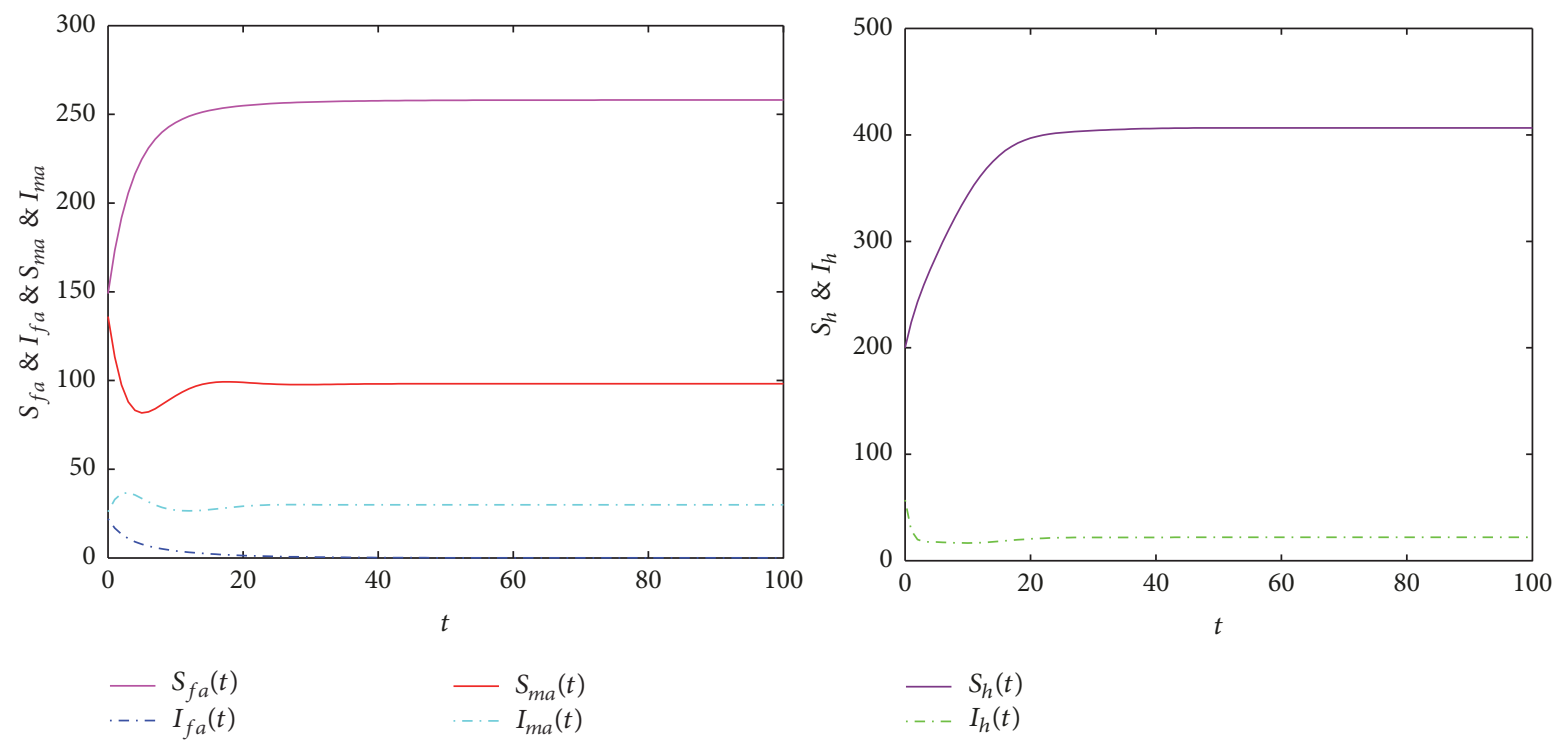

FIGURE 3: If $R_{01}=0.82949309<1$ and $R_{02}=1.83929618>1$, the time-variation diagram of system (25) state variables is shown.

Example 1. Taking parameters $A_{a}=80, \beta_{a}=0.0018, d_{a}=$ $0.17, \alpha_{a}=0.25, a=0.14, \beta_{m}=0.0027, d_{m}=0.2, \alpha_{m}=$ $0.35, A_{h}=50, \beta_{h}=0.0018, d_{h}=0.069, \alpha_{h}=0.63$, and $r=$ 0.301 , Figure 2 shows the time-variation diagram of system (25) state variables. It is found that if $R_{0}<1$, the disease-free equilibrium $E^{0}$ is globally asymptotically stable.

Example 2. Taking parameters $A_{a}=80, \beta_{a}=0.0018$, $d_{a}=0.17, \alpha_{a}=0.25, a=0.14, \beta_{m}=0.0056, d_{m}=0.2$, $\alpha_{m}=0.35, A_{h}=50, \beta_{h}=0.0018, d_{h}=0.069, \alpha_{h}=0.63$, and $r=0.301$, as shown in Figure 3 , it is found that if $R_{01}<1, R_{02}>1$, the boundary equilibrium $E^{*}$ is globally asymptotically stable.
Example 3. Taking parameters $A_{a}=80, \beta_{a}=0.0045, d_{a}=$ $0.17, \alpha_{a}=0.25, a=0.14, \beta_{m}=0.0056, d_{m}=0.2, \alpha_{m}=$ $0.35, A_{h}=50, \beta_{h}=0.0018, d_{h}=0.069, \alpha_{h}=0.63$, and $r=$ 0.301 , Figure 4 shows the time-variation diagram of system (25) state variables. It is found that if $R_{01}>1, R_{02}>1$, the endemic equilibrium $E^{* *}$ is globally asymptotically stable.

Example 4. Taking parameters $A_{a}=80, \beta_{a}=0.0045, d_{a}=$ $0.17, \alpha_{a}=0.25, a=0.14, \beta_{m}=0.0056, d_{m}=0.2, \alpha_{m}=0.35$, $A_{h}=50, d_{h}=0.069, \alpha_{h}=0.63$, and $r=0.301$ and letting $\beta_{h}=0.00005,0.0018,0.02$, Figure 5 shows the curve-trend diagram of $I_{h}$ with time. It is found that $I_{h}$ will increase with the increase of $\beta_{h}$. 

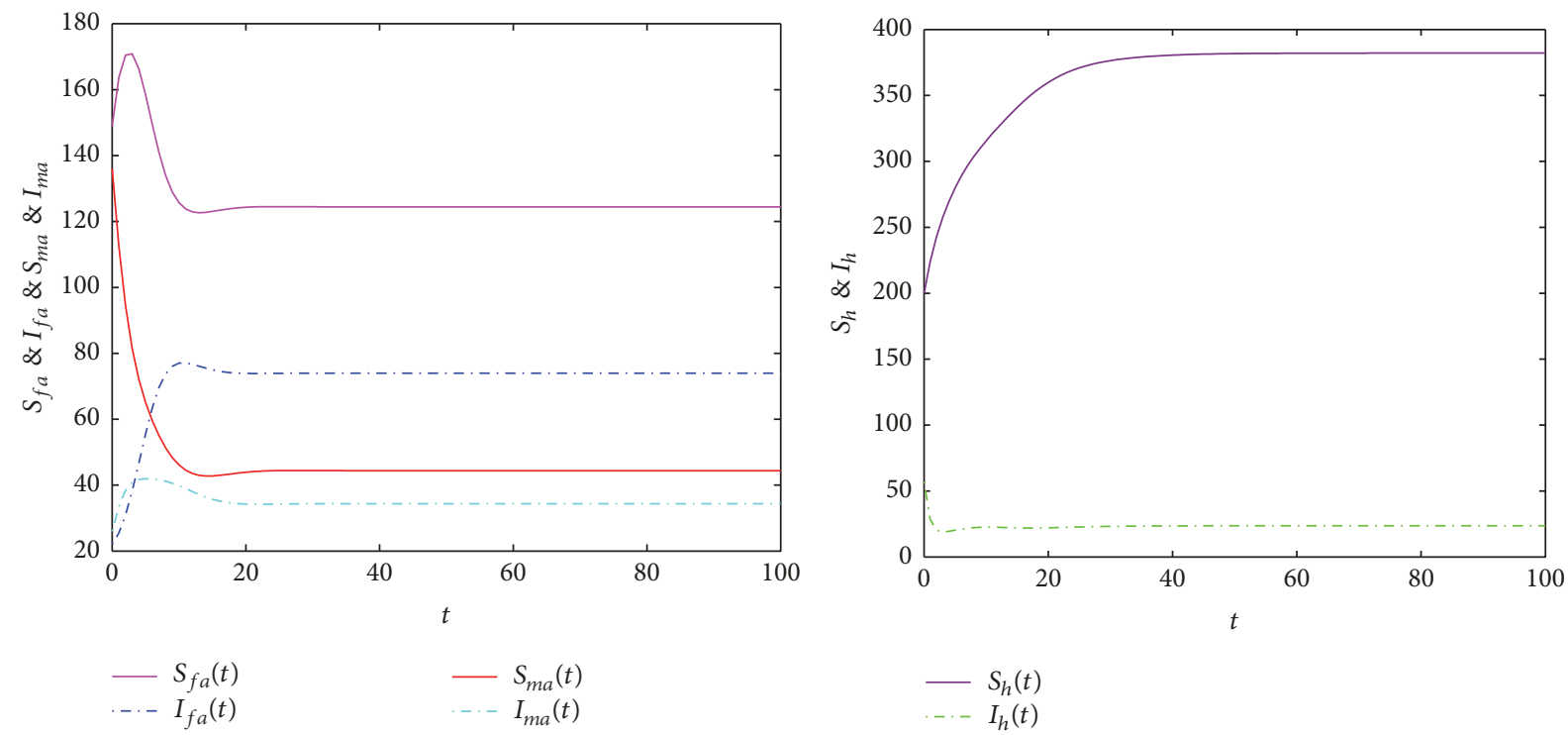

FIGURE 4: If $R_{01}=2.07373271>1$ and $R_{02}=1.83929618>1$, the time-variation diagram of system (25) state variables is shown.

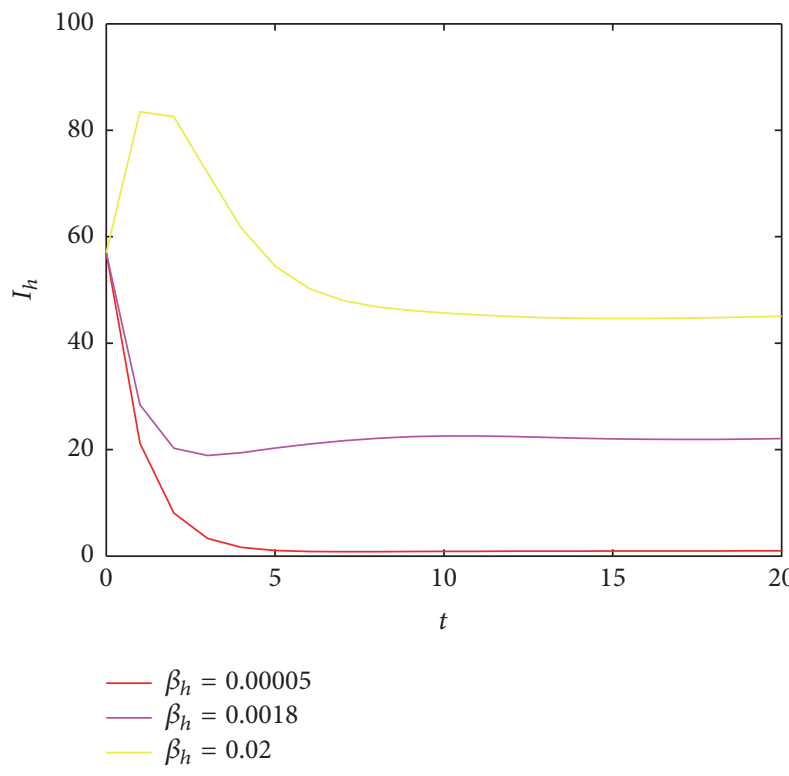

Figure 5: The curve-trend diagram of $I_{h}$ with time, when $\beta_{h}$ has different parameter values.

\section{Discussion}

Avian influenza infectious diseases caused by influenza virus can quickly spread in areas such as farms and markets. In this paper, an SI-SI-SIR dynamic model of avian influenza $\mathrm{A}(\mathrm{H} 7 \mathrm{~N} 9)$ is established by combining human and poultry. We get the basic reproduction number $R_{0}$; it is the threshold which is endemic or not. If $R_{0}<1$, there is only the disease-free equilibrium $E^{0}$, and it is globally asymptotically stable, which implies that the disease dies out. If $R_{01}<$ $1, R_{02}>1$, at this time $R_{0}>1$, there is the unique boundary equilibrium $E^{*}$, which is globally asymptotically stable; namely, the disease will be sustained and lead to epidemic disease eventually. If $R_{01}>1, R_{02}<1$, at this time $R_{0}>1$, there is no positive equilibrium; that is, the disease spreads in farms and it does not spread in markets, people will not be infected by the virus, and the disease will not be popular. If $R_{01}>1, R_{02}>1$, at this time $R_{0}>1$, there is the endemic equilibrium $E^{* *}$, which is globally asymptotically stable; that is, the disease will spread. From the above analysis, we can see that if artificial measures are taken to reduce the basic reproduction number to small enough value in the transmission system of avian influenza A(H7N9), the global stability point of the propagation dynamics process exists. Raise $\alpha_{a}$ and $\alpha_{m}$ by killing infected poultry or reduce $\beta_{a}, \beta_{m}$, and $\beta_{h}$ by closing farms and markets. Both of the two methods can reduce $R_{0}$ or $I_{h}$, so as to control the occurrence and development of diseases.

\section{Data Availability}

No data were used to support this study.

\section{Conflicts of Interest}

The authors declare no competing financial interests.

\section{Acknowledgments}

This work is supported by the National Sciences Foundation of China (11314091, 11501339) and the Youth Science Fund of Shanxi Province (201601D021015).

\section{References}

[1] D. J. Alexander, "A review of avian influenza in different bird species," Veterinary Microbiology, vol. 74, no. 1-2, pp. 3-13, 2000.

[2] E. C. J. Claas, A. D. M. E. Osterhaus, R. van Beek et al., "Human influenza A H5N1 virus related to a highly pathogenic avian influenza virus," The Lancet, vol. 351, no. 9101, pp. 472-477, 1998. 
[3] WHO, Cumulative number of confirmed human cases of avian influenza $A(\mathrm{H} 5 \mathrm{~N} 1)$ reported to $W H O$, World Health Organization, 2017, http://www.who.int/influenza/human_animal_ interface/H5N1_cumulative_table_archives/en/.

[4] WHO, Number of confirmed human cases of avian influenza A (H7N9) reported to World Health Organization, World Health Organization, 2013, http://www.who.int/influenza/human_animal_ interface/influenza_h7n9/08_ReportWebH7N9Number.pdf.

[5] R. Gao, B. Cao, and Y. Hu, "Human infection with a novel avianorigin influenza A (H7N9) virus," The New England Journal of Medicine, vol. 368, no. 20, pp. 1888-1897, 2013.

[6] T. Watanabe, M. Kiso, S. Fukuyama et al., "Characterization of H7N9 influenza A viruses isolated from humans," Nature, vol. 501, no. 7468, pp. 551-555, 2013.

[7] S. Iwami, Y. Takeuchi, and X. Liu, "Avian-human influenza epidemic model," Mathematical Biosciences, vol. 207, no. 1, pp. $1-25,2007$.

[8] S. Che, Y. Xue, and L. Ma, "The Stability of Highly Pathogenic Avian Influenza Epidemic Model with Saturated Contact Rate," Applied Mathematics, vol. 05, no. 21, pp. 3365-3371, 2014.

[9] Z. Liu and C.-T. Fang, "A modeling study of human infections with avian influenza A H7N9 virus in mainland China," International Journal of Infectious Diseases, vol. 41, pp. 73-78, 2015.

[10] Y. Chen and Y. Wen, "Global dynamic analysis of a H7N9 avian-human influenza model in an outbreak region," Journal of Theoretical Biology, vol. 367, pp. 180-188, 2015.

[11] S. Liu, S. Ruan, and X. Zhang, "Nonlinear dynamics of avian influenza epidemic models," Mathematical Biosciences, vol. 283, pp. 118-135, 2017.

[12] P. van den Driessche and J. Watmough, "Reproduction numbers and sub-threshold endemic equilibria for compartmental models of disease transmission," Mathematical Biosciences, vol. 180, pp. 29-48, 2002.

[13] J. P. LaSalle, "Stability theory for ordinary differential equations," Journal of Differential Equations, vol. 4, pp. 57-65, 1968.

[14] J. P. LaSalle, "The stability of dynamical systems," in Proceedings of the Regional Conference Series in Applied Mathematics, SIAM, Philadelphia, Penn, USA, 1976.

[15] Z. Wang, J. Cao, Z. Duan, and X. Liu, "Synchronization of coupled Duffing-type oscillator dynamical networks," Neurocomputing, vol. 136, pp. 162-169, 2014.

[16] Z. Wang, G. Jiang, W. Yu, W. He, J. Cao, and M. Xiao, "Synchronization of coupled heterogeneous complex networks," Journal of The Franklin Institute, vol. 354, no. 10, pp. 4102-4125, 2017. 


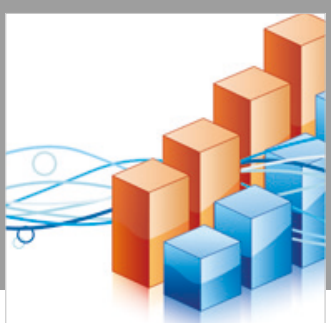

Advances in

Operations Research

\section{-n-m}
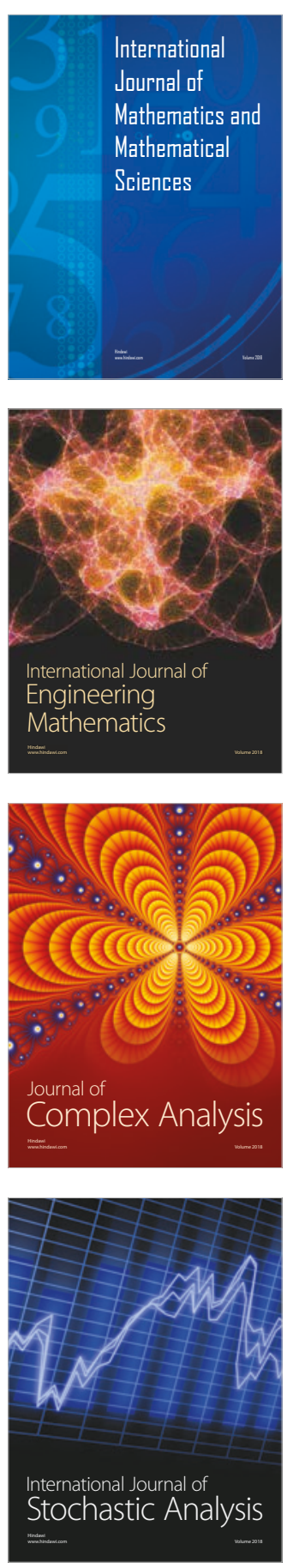
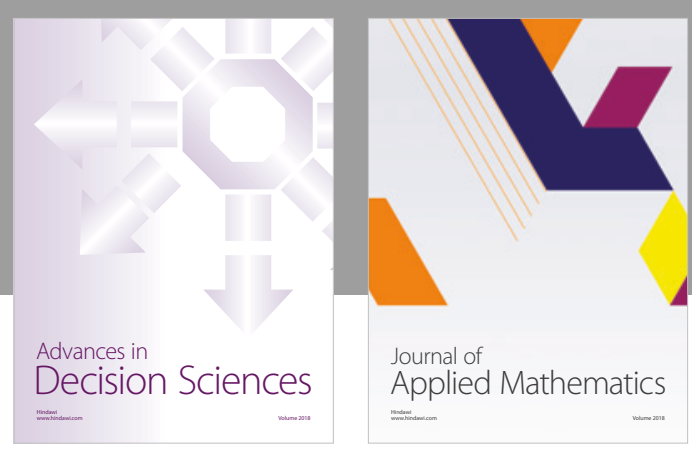

Journal of

Applied Mathematics
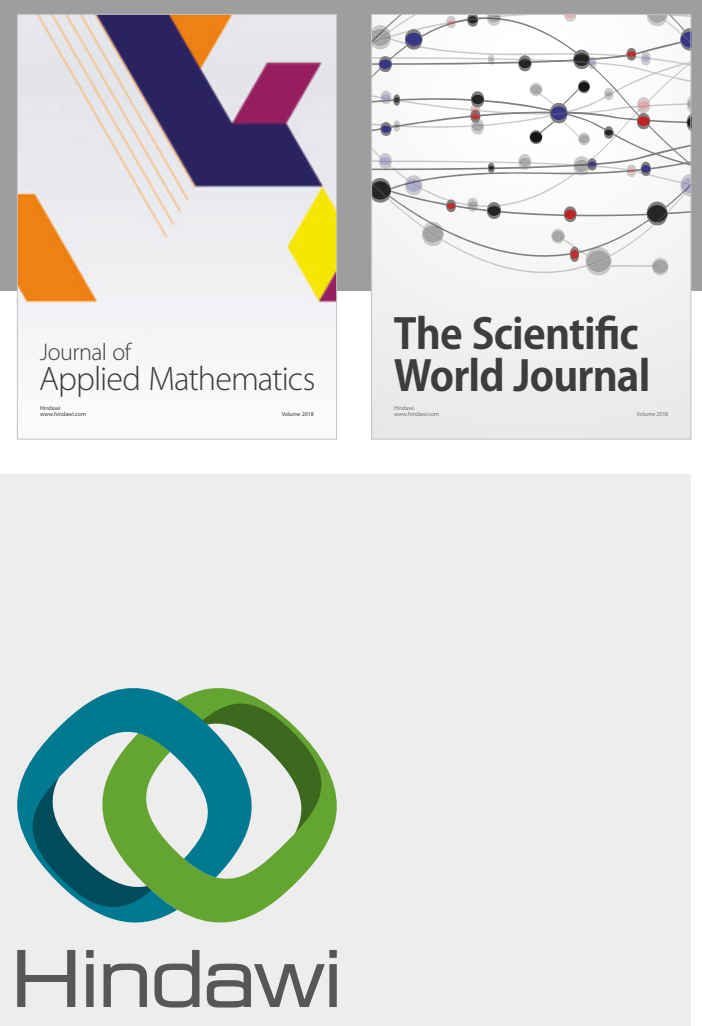

Submit your manuscripts at

www.hindawi.com

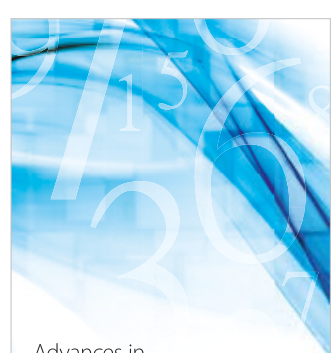

Advances in
Numerical Analysis
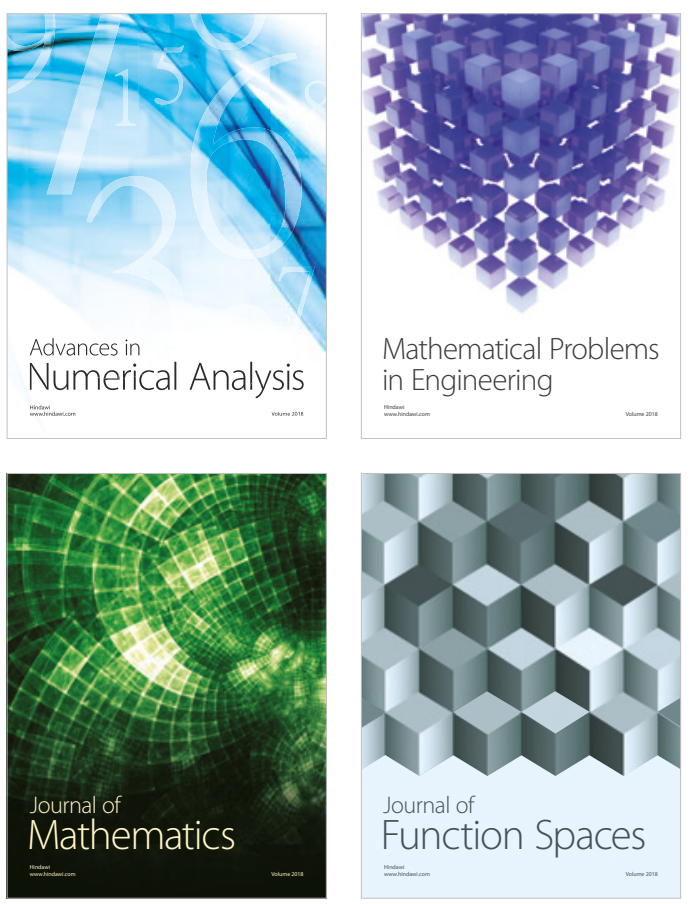

Mathematical Problems in Engineering

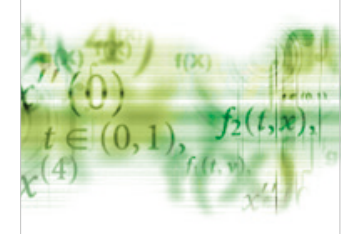

International Journal of

Differential Equations

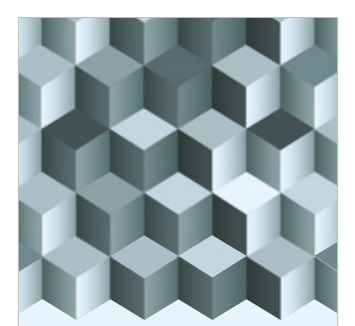

Journal of

Function Spaces

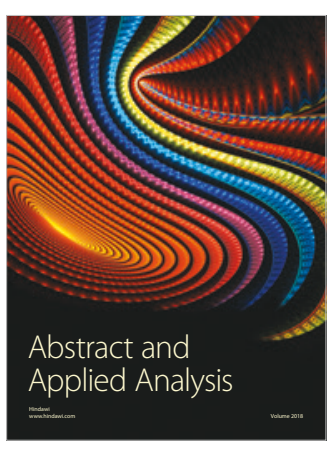

The Scientific

World Journal

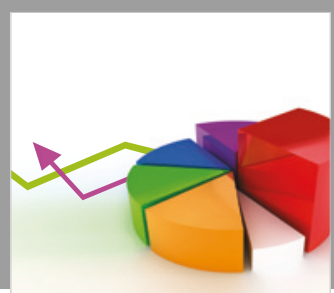

Journal of

Probability and Statistics
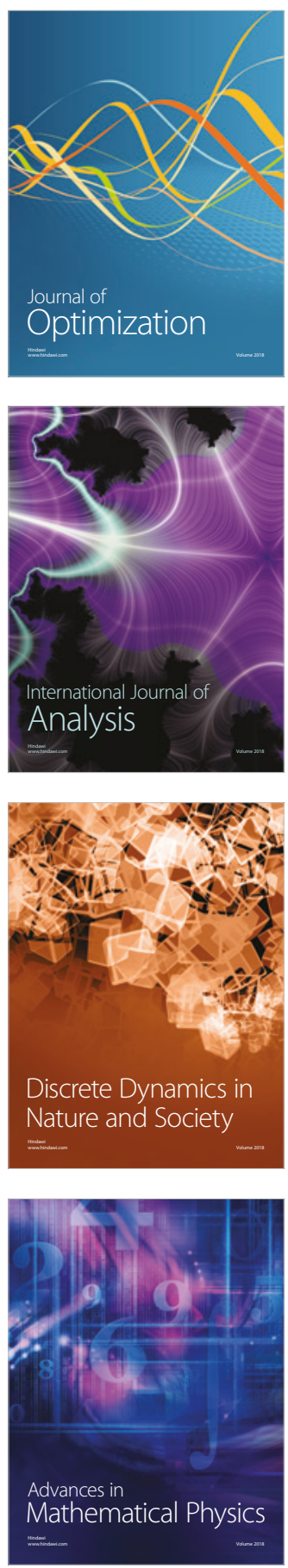